ISSN 1112-9867

Available online at

http://www.jfas.info

\title{
COMBINING ABILITY AND GENE ACTION ESTIMATES FOR SOME YIELD ATTRIBUTES IN DURUM WHEAT (Triticum turgidum L. var. durum)
}

\author{
A. Hannachi ${ }^{1,2}$, Z. Fellahi ${ }^{1,3}$, A. Rabti ${ }^{1}$, A. Guendouz ${ }^{2}$ and H. Bouzerzour ${ }^{4}$ \\ ${ }^{1}$ Département d'Agronomie, Fac. Sci. Nat. Vie, UFA Sétif 1, Algérie. \\ ${ }^{2}$ Institut National de la Recherche Agronomique (INRAA), Unité de Recherche de Sétif, Algérie. \\ ${ }^{3}$ Département d'Agronomie, Fac. Sci. Nat. Vie Sci. Univers, UMBI Bordj Bou Arréridj, Algérie. \\ ${ }^{4}$ Département d'Ecologie et Biologie Végétale, Fac. Sci. Nat. Vie, Laboratoire VRBN, UFA Sétif 1, Algérie.
}

Received: 22 January 2017 / Accepted: 19 August 2017 / Published online: 01 September 2017

\begin{abstract}
Combining ability and gene action of a set of durum wheat characters were studied using $6 \mathrm{x}$ 6 half diallel crosses. Results revealed that general (GCA) and specific (SCA) combining ability mean squares were significant for all studied traits, suggesting the importance of both additive and nonadditive components in the inheritance of these traits. Baker ratio indicated the predominance of additive gene effects for the majority of traits studied except grain yield. From the GCA effect, it was observed that none of the six parents was a good general combiner for all yield components. Among the fifteen crosses, Zenati-Bouteille/Flamengo x Gta/durum followed by Waha x Ofanto, Waha x Mexicali 75 and Ofanto x Guemgoum Rkhem expressed the greatest positive SCA effect for grain yield. Days to heading and spikes number index could be used as an indirect selection criterion for better grain yield. Therefore, selecting early heading genotypes having high fertile tillers number could improve grain yield.
\end{abstract}

Keywords: durum wheat; diallel cross; GCA; SCA; yield.

\footnotetext{
*Author Correspondence, e-mail: abderhannachi@yahoo.fr doi: http://dx.doi.org/10.4314/jfas.v9i3.17
} 


\section{INTRODUCTION}

Dans le monde, le blé dur (Triticum turgidium L. var. durum) est cultivé sur environ 17 millions d'hectares. Le bassin méditerranéen est la plus grande zone de production et de consommation du blé tout comme où elle est, aussi, la principale culture. Sa conduite est réalisée sous conditions pluviales ce qui l'expose souvent aux effets des divers stress tant biotiques qu'abiotiques générés par la dominance, en fin de cycle, par les hautes températures et le déficit hydrique [1]. L'amélioration des rendements de cette espèce peut être réalisée à travers 1'adoption des techniques de cultures adéquates et/ou par l'amélioration génétique pour le développement de génotypes performants et adaptés à la variation des milieux de production [2]. Le rendement en grains est un caractère à hérédité complexe et il est fortement affecté par l'environnement [3]. Il est la résultante de l'interaction de plusieurs caractéristiques morpho-physio-agronomiques, dont les composantes du rendement sont les plus déterminantes [4]. L'amélioration des caractères complexes comme le rendement est accomplie via l'amélioration des différentes composantes. Ces caractères montrent une hérédité moins complexe et sont liés au rendement [5]. Ainsi pour parvenir à améliorer de tels caractères, le sélectionneur se doit de connaître la nature des actions géniques impliquées dans leur contrôle génétique [6]. En effet seule l'action génique de nature additive et les interactions de nature additive $\mathrm{x}$ additive sont exploitables en sélection chez les espèces autogames comme le blé [7]. De telles informations, ainsi que les relations entre les composantes et le rendement, déterminent la méthode de sélection à déployer pour développer des variétés de blé à haut potentiel de rendement $[8,9]$. Le dispositif diallèle est employé par les sélectionneurs pour déterminer les effets d'aptitudes à la combinaison [10].

L'aptitude générale à la combinaison est la capacité d'un génotype à transmettre ses caractéristiques désirables à sa descendance. Cette capacité est largement déterminée par les actions de nature additive. Par contre l'aptitude spécifique à la combinaison est la capacité de certaines combinaisons hybrides à montrer des performances significativement plus élevées ou plus faibles que celles prédites sur la base de la performance moyenne des parents. Des résultats de diverses recherches suggèrent que la sélection précoce est effective pour les caractères à hérédité simple et qui sont sous contrôle génétique de nature additive. Par contre, 
elle doit être retardée pour les caractères complexes qui sont sous contrôle polygénique de nature non additive [11]. Des effets d'AGC et d'ASC significatifs, avec prédominance de la dominance sont observés pour la biomasse, le rendement, le poids des épis et le nombre d'épis, suggérant de retarder la sélection de ces caractères [11]. Par contre, la prépondérance des effets additifs est rapportée par Kashif et Khaliq [12], Mahmood et al. [13] et Rathod et al. [14] lesquels suggèrent que des croisements simples soient réalisés entre parents de bonnes valeurs propres, suivis de la sélection précoce, pour isoler des lignées de bonnes valeurs pour les caractères ciblés. La présence d'effets additifs et de dominance est rapportée par Rabbani et al. [15], Farooq et al. [16], Fellahi et al. [2, 8, 17]. L'objectif de cette investigation est de déterminer les aptitudes à la combinaison, le mode d'action des gènes, l'héritabilité et l'association génétique entre les caractères agronomiques des populations $F_{2}$ générées à partir d'un plan de croisement en demi-diallèle entre six génotypes de blé dur.

\section{EQUIPEMENTS ET METHODES}

L'expérimentation a été conduite sur le site expérimental de l'unité de recherche de l'INRAA, Station de Sétif $\left(36^{\circ} 15^{\prime} \mathrm{N}\right.$ et $5^{\circ} 37^{\prime}$ E, $\left.981 \mathrm{~m}\right)$ au cours de la campagne agricole 2013/14. Le matériel végétal est constitué de quinze populations $\mathrm{F}_{2}$ issues d'un croisement demi-diallèle (6 $\mathrm{x}$ 6) réalisé entre six variétés de blé dur [8]. Ces variétés sont Waha $\left(\mathrm{P}_{1}\right)$, Zenati-Bouteille/Flamengo $\left(\mathrm{P}_{2}\right)$, Mexicali ${ }_{75}\left(\mathrm{P}_{3}\right)$, Ofanto $\left(\mathrm{P}_{4}\right)$, Gta/durum $\left(\mathrm{P}_{5}\right)$ et Guemgoum Rkhem $\left(\mathrm{P}_{6}\right)$. Les parents et les populations $\mathrm{F}_{2}$ ont été semés dans un dispositif en blocs complètement randomisés avec trois répétitions. La parcelle élémentaire est constituée d'un rang de $10 \mathrm{~m}$ de long, avec un espace inter rangs de $30 \mathrm{~cm}$. L'espace entre graines sur le rang est de $15 \mathrm{~cm}$. Les notations et mesures ont porté sur la détermination de la teneur en chlorophylle (Chl, Spad), la date d'épiaison (DE, jours), la hauteur de la plante (HT, cm), la longueur de l'épi (LE, cm), le nombre d'épis par plante (NE), le nombre de grains par épi (NGE), le poids de mille grains (PMG, g), le rendement en grains par plante (RDT, g) et l'indice de récolte (IR, \%). Les données collectées sur les parents et leurs hybrides ont été soumises à une analyse de la variance selon Steel et Torrie [18] pour déterminer l'effet génotype. Les caractères, qui montrent un effet génotype significatif sont soumis à une 
analyse génétique selon la méthode II, modèle I de Griffing [19].

La variance additive $\left(\sigma^{2}{ }_{A}\right)$, représentant la proportion de la variation génétique due aux effets des gènes additifs, et la variance de dominance $\left(\sigma_{D}^{2}\right)$, qui exprime la variation génétique non additive, sont déterminées par : $\sigma_{A}^{2}=2 \sigma_{A G C}^{2} \quad, \sigma_{D}^{2}=\sigma_{A S C}^{2}$

Les variances [génétique $\left(\sigma_{G}^{2}\right)$, phénotypique $\left(\sigma_{P}^{2}\right)$ et environnementale $\left(\sigma_{E}^{2}\right)$ ] sont calculées par: $\sigma_{G}^{2}=\sigma_{A}^{2}+\sigma_{D}^{2}, \sigma_{P}^{2}=\sigma_{G}^{2}+\sigma_{E}^{2}, \sigma_{E}^{2}=C M_{\text {erreur }}$

Le ratio génétique de Baker $(R G)$ [20] a été calculé pour déterminer le mode d'action des gènes :

$$
\mathrm{RG}=2 \sigma^{2}{ }_{A G C} /\left(2 \sigma^{2}{ }_{A G C}+\sigma^{2}{ }_{A S C}\right)
$$

Où : $\sigma^{2}{ }_{A G C}$ et $\sigma^{2}{ }_{A S C}$ sont respectivement les variances de l'AGC et de l'ASC.

Un ratio proche de l'unité suggère la prépondérance des effets géniques de nature additive. Des valeurs proches de zéro $(0 \leq \mathrm{RG} \leq 0.5)$ expriment, par contre, la dominance des effets géniques non additifs.

L'héritabilité au sens large $\left(h_{b}^{2}\right)$ et au sens étroit $\left(h_{n}^{2}\right)$ sont calculées par [21]:

$$
h_{b}^{2}=\frac{\sigma_{G}^{2}}{\sigma_{P}^{2}} \quad ; \quad h_{n}^{2}=\frac{\sigma^{2} A}{\sigma_{P}^{2}}
$$

Les valeurs prises par l'héritabilité sont classées comme faibles $(<0.30)$, modérées $(0.30-0.60)$ et élevées $(>0.60)$.

Les analyses statistiques sont faites par le logiciel GENES [22].

\section{RESULTATS ET DISCUSSION}

\subsection{Analyse de la variance et performances moyennes}

L'analyse de la variance a révélé un effet génotype hautement significatif suggérant la présence de la variabilité pour l'ensemble des caractères étudiés, justifiant ainsi l'analyse génétique (Table 1). Les valeurs moyennes des différents caractères mesurés des parents et des $\mathrm{F}_{2}$ sont présentées dans la table 2. Chez les parents, Guemgoum Rkhem ( $\left.\mathrm{P}_{6}\right)$ s'est distingué par des valeurs moyennes élevées pour la date d'épiaison (146.00 jours), la hauteur de la végétation $(101.28 \mathrm{~cm})$, la longueur de l'épi $(8.03 \mathrm{~cm})$ et le poids de mille grains $(63.63$ g). Cette variété a, par contre, affiché les plus faibles valeurs de la teneur en chlorophylle (26.22 unité Spad), la fertilité des épis (31.59 grains) et l'indice de récolte $(30.44 \%)$. La 
variété Ofanto $\left(\mathrm{P}_{4}\right)$ a montré des performances pour la teneur en chlorophylle (41.76 unité Spad) et l'indice de récolte (43.59\%).

Table 1. Carrés moyens de l'analyse de la variance des caractères mesurés en $F_{2}$.

\begin{tabular}{|l|r|r|r|r|r|r|r|r|r|r|}
\hline Source & \multicolumn{1}{l|}{ DF } & \multicolumn{1}{l|}{ Chl } & \multicolumn{1}{l|}{ DE } & HT & LE & NE & \multicolumn{1}{l|}{ NGE } & PMG & \multicolumn{1}{l|}{ RDT } & \multicolumn{1}{l|}{ IR } \\
\hline Blocs & 2 & 4.8 & 6.3 & 0.5 & 0.06 & 0.9 & 8.9 & 6.3 & 5.7 & 0.66 \\
\hline Génotypes & 20 & $60.6^{* *}$ & $17.8^{* *}$ & $400.9^{* *}$ & $1.08^{* *}$ & $1.6^{* *}$ & $148.2^{* *}$ & $173.7 * *$ & $5.9^{* *}$ & $35.64 * *$ \\
\hline Erreur & 40 & 7.4 & 1.0 & 1.6 & 0.09 & 0.18 & 15.3 & 5.7 & 1.7 & 3.19 \\
\hline CV (\%) & & 7.86 & 0.76 & 1.81 & 3.90 & 8.40 & 8.17 & 5.49 & 13.22 & 4.44 \\
\hline
\end{tabular}

Chl: Teneur en chlorophylle (unité Spad); DE: Date d'épiaison (jours); HT: Hauteur de la plante (cm); NE: Nombre d'épi par plante; NGE: Nombre de grains par épi; PMG: Poids de mille grains (g); RDT: Rendement en grains par plante (g); IR: Indice de récolte (\%); ** : Effet significatif au seuil de 1\%; CV: Coefficient de variation (\%).

Les moyennes les plus élevées du nombre d'épis par plante (5.70 épis), du nombre de grains par épi (58.22 graines) et du rendement en grains (12.61 g) sont notées, respectivement chez Waha $\left(\mathrm{P}_{1}\right)$, Gta/durum $\left(\mathrm{P}_{5}\right)$ et Mexicali $_{75}\left(\mathrm{P}_{3}\right)$. D'autre part, Waha $\left(\mathrm{P}_{1}\right)$, Zenati Bouteille/Flamengo $\left(\mathrm{P}_{2}\right)$ et Ofanto $\left(\mathrm{P}_{4}\right)$ sont les parents les plus précoces avec 136 jours, soit une différence de 10 jours relativement au cultivar le plus tardif, Guemgoum Rkhem $\left(\mathrm{P}_{6}\right)$. Zenati Bouteille/Flamengo $\left(\mathrm{P}_{2}\right)$ se distingue aussi par une hauteur $(60.43 \mathrm{~cm})$ et un épi $(6.49$ $\mathrm{cm}$ ) les plus courts. Le potentiel de tallage-épi le plus faible (5 épis/plante) est mesuré chez Ofanto $\left(\mathrm{P}_{4}\right)$ alors que le poids moyen de 1000 grains le plus faible $(36.47 \mathrm{~g})$ est exprimé chez Mexicali 75 .

Chez les populations $\mathrm{F}_{2}$, l'étude des valeurs moyennes montre que les meilleures valeurs sont variables, selon le caractère, et aucune population ne présente des valeurs élevées pour l'ensemble des caractères mesurés. Ainsi, la population Waha x Ofanto $\left(\mathrm{P}_{1} \times \mathrm{P}_{4}\right)$ présente les meilleures performances pour la teneur en chlorophylle (40.54 unité Spad), le rendement en grains $(12.61 \mathrm{~g})$ et l'indice de récolte $(46.54 \mathrm{~g})$. Mexicali 75 x Guemgoum Rkhem $\left(\mathrm{P}_{3} \times \mathrm{P}_{6}\right)$ se caractérise par sa tardivité (141.00 jours) et la longueur de l'épi $(9.02 \mathrm{~cm})$, alors que Waha x Guemgoum Rkhem $\left(\mathrm{P}_{1} \times \mathrm{P}_{6}\right)$, Mexicali 75 x Gta/durum $\left(\mathrm{P}_{3} \times \mathrm{P}_{5}\right)$ et Ofanto x Guemgoum Rkhem $\left(\mathrm{P}_{4} \times \mathrm{P}_{6}\right)$ expriment les meilleures performances pour la hauteur $(90.22 \mathrm{~cm})$, le nombre de grains par épi (60.06 graines) et le poids de mille grains (52.05 g). Globalement, les populations ayant Guemgoum Rkhem $\left(\mathrm{P}_{6}\right)$ comme géniteur sont intéressantes pour 
améliorer la hauteur de la plante, la longueur de l'épi et le poids de mille grains. Celles ayant Ofanto $\left(\mathrm{P}_{4}\right)$ comme parent sont intéressantes pour réduire la durée du cycle végétatif et améliorer la teneur en chlorophylle et le rendement en grains. Relativement aux valeurs moyennes observées chez les parents, les populations $\mathrm{F}_{2}$ présentent des améliorations notables des caractères mesurés (Table 2).

Table 2. Valeurs moyennes des caractères mesurés chez les parents et les populations $F_{2}$.

\begin{tabular}{|c|c|c|c|c|c|c|c|c|c|}
\hline Génotype & Chl & DE & HT & $\mathbf{L E}$ & NE & NGE & PMG & RDT & IR \\
\hline$P_{1}$ & 36.89 & 136.00 & 67.41 & 7.18 & 5.70 & 41.64 & 37.27 & 7.91 & 41.19 \\
\hline $\mathbf{P}_{2}$ & 38.30 & 136.00 & 60.43 & 6.49 & 5.27 & 36.92 & 37.20 & 7.78 & 38.69 \\
\hline $\mathbf{P}_{3}$ & 34.00 & 139.00 & 61.98 & 7.77 & 4.80 & 55.85 & 36.47 & 10.42 & 39.02 \\
\hline $\mathbf{P}_{4}$ & 41.76 & 136.00 & 61.57 & 7.10 & 5.00 & 45.60 & 50.77 & 8.84 & 43.59 \\
\hline $\mathbf{P}_{5}$ & 31.71 & 137.00 & 62.12 & 7.57 & 3.97 & 58.22 & 38.30 & 8.48 & 43.04 \\
\hline $\mathbf{P}_{6}$ & 26.22 & 146.00 & 101.28 & 8.03 & 4.17 & 31.59 & 63.63 & 8.25 & 30.44 \\
\hline $\mathbf{P}_{1} \times \mathbf{P}_{2}$ & 36.83 & 137.00 & 68.72 & 7.31 & 6.13 & 45.49 & 37.17 & 9.56 & 37.80 \\
\hline $\mathbf{P}_{1} \times \mathbf{P}_{3}$ & 39.45 & 136.33 & 66.73 & 7.68 & 6.27 & 50.28 & 38.89 & 12.21 & 41.73 \\
\hline $\mathbf{P}_{1} \times \mathbf{P}_{4}$ & 40.54 & 135.67 & 64.67 & 7.26 & 6.23 & 50.04 & 40.20 & 12.61 & 46.54 \\
\hline $\mathbf{P}_{1} \times \mathbf{P}_{5}$ & 37.60 & 136.67 & 67.52 & 7.67 & 5.97 & 48.68 & 37.50 & 10.45 & 41.45 \\
\hline $\mathbf{P}_{1} \times \mathbf{P}_{6}$ & 31.80 & 138.33 & 90.22 & 8.28 & 5.63 & 41.20 & 48.57 & 10.67 & 38.52 \\
\hline $\mathbf{P}_{2} \times \mathbf{P}_{3}$ & 30.55 & 138.67 & 63.43 & 7.13 & 5.13 & 45.47 & 38.40 & 8.92 & 39.10 \\
\hline $\mathbf{P}_{2} \times \mathbf{P}_{4}$ & 39.11 & 136.67 & 67.42 & 6.92 & 4.33 & 47.18 & 45.60 & 9.96 & 43.19 \\
\hline $\mathbf{P}_{2} \times \mathbf{P}_{5}$ & 29.11 & 136.67 & 67.73 & 7.45 & 5.90 & 50.60 & 37.33 & 11.54 & 39.43 \\
\hline $\mathbf{P}_{2} \times \mathbf{P}_{6}$ & 28.44 & 140.67 & 88.42 & 7.95 & 4.00 & 46.56 & 54.22 & 10.71 & 36.75 \\
\hline $\mathbf{P}_{3} \times \mathbf{P}_{4}$ & 37.66 & 136.00 & 64.72 & 7.73 & 5.07 & 47.75 & 42.45 & 11.22 & 41.71 \\
\hline $\mathbf{P}_{3} \times \mathbf{P}_{5}$ & 32.23 & 139.67 & 61.55 & 7.84 & 5.33 & 60.06 & 36.69 & 8.98 & 43.14 \\
\hline $\mathbf{P}_{3} \times \mathbf{P}_{6}$ & 30.75 & 141.00 & 86.87 & 9.02 & 4.47 & 57.01 & 51.20 & 9.76 & 36.04 \\
\hline $\mathbf{P}_{4} \times \mathbf{P}_{5}$ & 40.01 & 137.00 & 66.82 & 7.62 & 4.63 & 56.18 & 42.40 & 10.38 & 43.59 \\
\hline $\mathbf{P}_{4} \times \mathbf{P}_{6}$ & 36.20 & 138.00 & 82.73 & 8.76 & 5.03 & 44.50 & 52.05 & 12.07 & 40.73 \\
\hline $\mathbf{P}_{5} \times \mathbf{P}_{6}$ & 31.59 & 139.33 & 75.43 & 8.28 & 4.30 & 48.01 & 50.37 & 10.40 & 38.57 \\
\hline Moy. Parents & 34.81 & 138.33 & 69.13 & 7.36 & 4.82 & 44.97 & 43.94 & 8.61 & 39.33 \\
\hline Moy. F $F_{2}$ & 34.79 & 137.85 & 72.20 & 7.79 & 5.23 & 49.27 & 43.54 & 10.63 & 40.55 \\
\hline Ppds $_{5 \%}$ & 5.56 & 1.79 & 5.72 & 0.61 & 0.88 & 8.89 & 5.24 & 2.56 & 3.80 \\
\hline
\end{tabular}

Chl: Teneur en chlorophylle (unité Spad); DE: Date d'épiaison (jours); HT: Hauteur de la plante (cm); NE: Nombre d'épi par plante; NGE: Nombre de grains par épi; PMG: Poids de mille grains (g); RDT: Rendement en grains par plante (g); IR: Indice de récolte (\%).

\subsection{Analyse des aptitudes à la combinaison et l'héritabilité}

Les carrés moyens des AGC et ASC sont donnés dans la table 3. Les effets d'AGC et ASC des variables étudiées sont significatifs pour la majorité des caractères mesurés. Ces résultats 
indiquent que les actions géniques de nature additive et non additive sont impliquées dans l'expression de ces caractères à l'exception de l'AGC du rendement et l'ASC du poids de mille grains pour lesquels les effets sont non significatifs. Le ratio génétique de Baker pour la teneur en chlorophylle, la date d'épiaison, la hauteur, la longueur de l'épi, le nombre d'épi, le nombre de grains par épi, le poids de mille grains et l'indice de récolte indique la prépondérance des effets additifs dans le contrôle génétique de ces caractères (Table 3 ). Par conséquent il est suggéré que la sélection précoce pour l'amélioration de ces caractères serait efficace. D’ailleurs, des résultats similaires ont été rapportés par Adel et Ali [5], Iqbal et al. [23], Shahid et al. [24] et Bouzerzour et Djekoun [25]. La prépondérance des effets de dominance pour le rendement en grains par plant suggère la complexité du système génique impliqué dans l'expression de ce caractère pour lequel la sélection doit être tardive.

L'héritabilité au sens large est élevée pour l'ensemble des caractères étudiés à l'exception du rendement en grains pour lequel ce paramètre génétique est modéré (Table 6). Les valeurs prises par l'héritabilité au sens étroit sont faibles pour le rendement en grains, moyennes pour la teneur en chlorophylle des feuilles, la longueur et le nombre d'épis et le nombre de grains par épi, et élevées pour la date d'épiaison, la hauteur de la végétation et le poids de mille grains indiquant une réponse élevée à la sélection pour ces caractères (Table 6). Selon Falconer [26] et Dabholkar [27], l'héritabilité au sens étroit mesure l'ampleur de la variation génotypique qui est principalement responsable de la modification de la composition génétique de la population par sélection. De plus, l'héritabilité au sens étroit est directement proportionnelle à la variance génétique additive et est maximale pour les caractères contrôlés par des effets additifs que de dominance [28]. Des valeurs élevées de l'héritabilité ont été rapportées, pour ces mêmes caractères, par plusieurs chercheurs dont Riaz et al. [29] et Ali et al. [30]. La date d'épiaison, la hauteur de la végétation et le poids de mille grains pourraient être utiles comme des critères de sélection précoce des génotypes d'élite à l'intérieur de ce matériel génétique en ségrégation. 
Table 3. Analyse de la variance des aptitudes générale (AGC) et spécifique (ASC) à la combinaison, l'héritabilité et le ratio de Baker des caractères mesurés en $\mathrm{F}_{2}$.

\begin{tabular}{|l|r|r|r|r|r|r|r|r|r|r|}
\hline Source & ddl & \multicolumn{1}{l}{ Chl } & DE & HT & LE & NE & NGE & \multicolumn{1}{l|}{ PMG } & \multicolumn{1}{l|}{ RDT } & \multicolumn{1}{l|}{ IR } \\
\hline AGC & 5 & $192.19^{* *}$ & $59.81^{* *}$ & $1491.34^{* *}$ & $3.13^{* *}$ & $4.02^{* *}$ & $414.33^{* *}$ & $663.60^{* *}$ & $2.69^{\text {ns }}$ & $119.23^{* *}$ \\
\hline ASC & 15 & $16.84^{*}$ & $3.82^{* *}$ & $37.50^{* *}$ & $0.39^{* *}$ & $0.89^{* *}$ & $59.59^{* *}$ & $10.47^{\text {ns }}$ & $7.09^{* *}$ & $7.78^{*}$ \\
\hline Erreur & 40 & 7.47 & 1.10 & 1.67 & 0.09 & 0.18 & 15.39 & 5.75 & 1.77 & 3.19 \\
\hline $\boldsymbol{\sigma}^{\mathbf{2}}{ }_{\text {AGC }}$ & 23.71 & 7.43 & 186.35 & 0.39 & 0.50 & 51.15 & 82.71 & 0.26 & 14.77 \\
\hline $\boldsymbol{\sigma}^{\mathbf{2}}$ ASC & 7.18 & 1.73 & 18.47 & 0.18 & 0.42 & 27.23 & 4.28 & 3.25 & 3.36 \\
\hline $\mathbf{A G C / A S C}$ & 11.4 & 15.7 & 39.8 & 8.0 & 4.5 & 7.0 & 63.4 & 0.4 & 15.3 \\
\hline $\mathbf{R G}$ & 0.87 & 0.90 & 0.95 & 0.81 & 0.70 & 0.79 & 0.97 & 0.14 & 0.90 \\
\hline $\mathbf{h}_{\mathbf{b}}^{\mathbf{2}}$ & 0.71 & 0.84 & 0.99 & 0.80 & 0.75 & 0.76 & 0.91 & 0.51 & 0.78 \\
\hline $\mathbf{h}_{\mathbf{n}}^{\mathbf{2}}$ & 0.59 & 0.70 & 0.90 & 0.56 & 0.44 & 0.53 & 0.88 & 0.02 & 0.67 \\
\hline
\end{tabular}

Chl: Teneur en chlorophylle (unité Spad); DE: Date d'épiaison (jours); HT: Hauteur de la plante (cm); NE: Nombre d'épi par plante; NGE: Nombre de grains par épi; PMG: Poids de mille grains (g); RDT: Rendement en grains par plante (g); IR: Indice de récolte (\%); ${ }^{* *}$ et $*$ : Effets significatifs au seuil de $1 \%$ et $5 \%$, respectivement; $\mathrm{h}^{2}{ }_{\mathrm{b}}$ : Héritabilité au sens large; $\mathrm{h}_{\mathrm{n}}^{2}$ : Héritabilité au sens étroit ; RG: ratio génétique de Baker.

\subsection{Analyse des effets des aptitudes à la combinaison}

La valeur et le signe des effets d'aptitude à la combinaison sont utiles à connaitre car ils servent d'aides à la sélection, dans les programmes d'amélioration, des parents qui combinent bien et des meilleures combinaisons hybrides pour les caractères d'intérêts [31]. Waha $\left(\mathrm{P}_{1}\right)$ et Ofanto $\left(\mathrm{P}_{4}\right)$ présentent un effet d'AGC positif et significatif pour la chlorophylle et un effet d'AGC négatif et significatif pour la durée de la phase végétative (Table 4). Ces variétés sont donc à privilégier comme géniteurs dans les cas où on cible l'amélioration du contenu en chlorophylle et la réduction de la durée de la phase végétative. Dans le cas où la sélection se fait dans le sens de l'allongement de la durée de la phase végétative Guemgoum Rkhem $\left(\mathrm{P}_{6}\right)$ est le géniteur à considérer en croisement car il présente un effet d'AGC significatif et de signe positif, de valeur 2.93 jours. Sur les hautes plaines orientales la sélection est faite dans le sens de la réduction de la durée de la phase végétative pour minimiser les effets des stress de fin de cycle en termes de réduction du rendement en grains [32].

Pour la hauteur de la végétation, Zenati-Bouteille/Flamengo $\left(\mathrm{P}_{2}\right)$, Mexicali ${ }_{75}\left(\mathrm{P}_{3}\right)$, Ofanto $\left(\mathrm{P}_{4}\right)$ et Gta/durum $\left(\mathrm{P}_{5}\right)$ présentent des effets d'AGC significatifs et de signes négatifs, à l'inverse de Guemgoum Rkhem $\left(\mathrm{P}_{6}\right)$ qui présente un effet d'AGC positif et significatif. Ces résultats suggèrent les parents qui combinent le mieux pour réduire ou augmenter la hauteur de la végétation. La hauteur d'une variété est une caractéristique désirable pour les agriculteurs, 
notamment ceux qui pratiquent l'association céréales-élevage ovin ainsi que ceux qui adoptent la technique du semis direct. Elle détermine la quantité de paille à prélever comme litière et celle des résidus à laisser en plein champ pour protéger le sol de l'érosion hydrique et éolienne. Elle a des effets positifs sur le rendement en grains, notamment par la quantité de sucres solubles stockés temporairement au niveau du col de l'épi et remobilisée vers le grain au cours de la période de remplissage. Sous conditions semi-arides, les risques de verse, souvent avancés pour justifier l'utilisation des variétés courtes, sont rarement observés même chez les variétés locales les plus hautes.

Table 4. Valeurs des effets de l'aptitude générale à la combinaison (AGC).

\begin{tabular}{|l|r|r|r|r|r|r|r|r|r|}
\hline Génotype & Chl & DE & HT & LE & NE & NGE & PMG & RDT & IR \\
\hline $\mathbf{P}_{\mathbf{1}}$ & $2.05^{* *}$ & $-1.24^{* *}$ & -0.82 & -0.14 & $0.73^{* *}$ & $-2.16^{*}$ & $-3.50^{* *}$ & 0.12 & $0.88^{*}$ \\
\hline $\mathbf{P}_{\mathbf{2}}$ & -0.37 & -0.53 & $-2.83^{* *}$ & $-0.49^{* *}$ & 0.03 & $-3.39^{* *}$ & $-2.30^{* *}$ & -0.52 & $-0.97^{*}$ \\
\hline $\mathbf{P}_{\mathbf{3}}$ & -0.62 & 0.47 & $-4.00^{* *}$ & $0.16^{*}$ & 0.01 & $4.50^{* *}$ & $-3.12^{* *}$ & 0.20 & -0.208 \\
\hline $\mathbf{P}_{\mathbf{4}}$ & $4.18^{* *}$ & $-1.32^{* *}$ & $-3.72^{* *}$ & $-0.15^{*}$ & -0.06 & 0.07 & $2.33^{* *}$ & 0.44 & $2.69^{* *}$ \\
\hline $\mathbf{P}_{\mathbf{5}}$ & -1.20 & -0.32 & $-4.49^{* *}$ & 0.04 & $-0.21^{*}$ & $5.46^{* *}$ & $-3.08^{* *}$ & -0.21 & $1.36^{* *}$ \\
\hline $\mathbf{P}_{\mathbf{6}}$ & $-4.05^{* *}$ & $2.93^{* *}$ & $15.87^{* *}$ & $0.56^{* *}$ & $-0.50^{* *}$ & -4.48 & $9.77^{* *}$ & -0.03 & $-3.74^{* *}$ \\
\hline $\mathbf{S E}$ & 0.51 & 0.195 & 0.241 & 0.06 & 0.08 & 0.731 & 0.447 & 0.25 & 0.333 \\
\hline
\end{tabular}

Chl: Teneur en chlorophylle (unité Spad); DE: Date d'épiaison (jours); HT: Hauteur de la plante (cm); NE: Nombre d'épi par plante; NGE: Nombre de grains par épi; PMG: Poids de mille grains (g); RDT: Rendement en grains par plante (g); IR: Indice de récolte $(\%) ; *$ et $*$ : Effets significatifs au seuil de $1 \%$ et $5 \%$, respectivement; SE: Erreur standard.

Suite à l'effet d'AGC significatif et positif, pour la longueur de l'épi, Guemgoum Rkhem $\left(\mathrm{P}_{6}\right)$ se présente comme un bon combineur pour l'amélioration de cette caractéristique. Zenati-Bouteille/Flamengo $\left(\mathrm{P}_{2}\right)$ et Ofanto $\left(\mathrm{P}_{4}\right)$ montrent, par contre, des effets d'AGC de signes négatifs pour cette caractéristique. Pour le nombre d'épi, Waha $\left(\mathrm{P}_{1}\right)$ est conseillé comme géniteur dans les situations où l'on cherche à améliorer ce caractère. Pour la fertilité de l'épi, Ofanto $\left(\mathrm{P}_{4}\right)$ et Gta/durum $\left(\mathrm{P}_{5}\right)$ présentent des effets d'AGC significatifs et positifs alors que Waha $\left(\mathrm{P}_{1}\right)$ et Zenati-Bouteille/Flamengo $\left(\mathrm{P}_{2}\right)$ présentent des effets d'AGC négatifs et significatifs. Pour le poids de mille grains, tous les parents ont montré des effets d'AGC significatifs. Néanmoins, seuls Guemgoum Rkhem $\left(\mathrm{P}_{6}\right)$ et Ofanto $\left(\mathrm{P}_{4}\right)$ présentent des effets d'AGC significatifs et positifs avec des valeurs d'AGC de 2.33 et 9.77, respectivement. Pour l'indice de récolte, Waha $\left(\mathrm{P}_{1}\right)$, Ofanto $\left(\mathrm{P}_{4}\right)$ et Gta/durum $\left(\mathrm{P}_{5}\right)$ présentent des effets d'AGC 
significatifs et positifs. A l'opposé, Zenati-Bouteille/Flamengo $\left(\mathrm{P}_{2}\right)$ et Guemgoum Rkhem $\left(\mathrm{P}_{6}\right)$ présentent des effets significatifs et négatifs. Pour le rendement en grains, aucun parent ne présente d'effet d'AGC significatif. Ces résultats suggèrent qu'il est difficile d'améliorer le rendement, en utilisant le jeu des parents croisés.

L'aptitude spécifique à la combinaison (ASC) est considérée comme un bon critère pour sélectionner les meilleures populations $\mathrm{F}_{2}$ pour un ou plusieurs caractères d'intérêts. Les valeurs de l'ASC indiquent que plusieurs populations $F_{2}$ se distinguent par des effets significatifs (Table 5). Ainsi, la population Zenati-Bouteille/Flamengo x Gta/durum $\left(\mathrm{P}_{2} \times \mathrm{P}_{5}\right)$ présente des effets d'ASC significatifs et positifs pour la hauteur de la végétation (3.74), le nombre d'épis par plante (0.97) et le rendement (2.21). Waha x Ofanto $\left(\mathrm{P}_{1} \times \mathrm{P}_{4}\right)$ présente aussi des effets d'ASC, négatif pour la hauteur de la végétation (-2.11) et positifs pour le rendement (2.00) et l'indice de récolte (2.77).

Table 5. Valeurs des effets de l'aptitude spécifique à la combinaison (ASC).

\begin{tabular}{|c|c|c|c|c|c|c|c|c|c|}
\hline Croisement & Chl & DE & HT & LE & NE & NGE & PMG & RDT & IR \\
\hline $\mathbf{P}_{\mathbf{1}} \times \mathbf{P}_{\mathbf{2}}$ & 0.34 & 0.78 & 1.05 & 0.27 & 0.258 & 3.01 & -0.587 & -0.10 & $-2.31 *$ \\
\hline $\mathbf{P}_{1} \times \mathbf{P}_{3}$ & 3.22 & -0.89 & 0.23 & -0.002 & 0.412 & -0.10 & 1.953 & $1.84^{*}$ & 0.856 \\
\hline $\mathbf{P}_{1} \times \mathbf{P}_{4}$ & -0.49 & 0.24 & $-2.11 * *$ & -0.12 & 0.45 & 4.09 & -2.199 & $2.00^{*}$ & $2.77 * *$ \\
\hline $\mathbf{P}_{1} \times \mathbf{P}_{5}$ & 1.96 & 0.24 & $1.52 *$ & 0.10 & 0.337 & -2.66 & 0.516 & 0.49 & -0.99 \\
\hline $\mathbf{P}_{1} \times \mathbf{P}_{6}$ & -1.01 & $-1.35^{*}$ & $3.85 * *$ & 0.17 & 0.292 & -0.20 & -1.263 & 0.53 & 1.18 \\
\hline $\mathbf{P}_{2} \times \mathbf{P}_{3}$ & $-3.26^{*}$ & 0.74 & -1.057 & -0.2 & -0.021 & -3.68 & 0.18 & -0.81 & 0.08 \\
\hline $\mathbf{P}_{2} \times \mathbf{P}_{4}$ & 0.50 & 0.53 & $2.65 * *$ & -0.11 & $-0.75 * *$ & 2.46 & 1.915 & -0.02 & 1.27 \\
\hline $\mathbf{P}_{2} \times \mathbf{P}_{5}$ & $-4.16^{*}$ & -0.47 & $3.74 * *$ & 0.23 & $0.97 * *$ & 0.49 & -0.933 & $2.21 * *$ & -1.15 \\
\hline $\mathbf{P}_{2} \times \mathbf{P}_{6}$ & -1.94 & 0.28 & $4.06^{* *}$ & 0.19 & $-0.64 *$ & $6.39 * *$ & $3.11^{*}$ & 1.21 & 1.26 \\
\hline $\mathbf{P}_{3} \times \mathbf{P}_{4}$ & -0.70 & -1.14 & 1.114 & 0.05 & 0.004 & $-4.86^{*}$ & -0.41 & 0.53 & -0.98 \\
\hline $\mathbf{P}_{3} \times \mathbf{P}_{5}$ & -0.75 & 1.53 & -1.277 & -0.024 & 0.43 & 2.06 & -0.75 & -1.06 & 1.79 \\
\hline $\mathbf{P}_{3} \times \mathbf{P}_{6}$ & 0.62 & -0.39 & $3.67 * *$ & 0.606 & -0.15 & $8.95 * *$ & 0.90 & -0.46 & -0.28 \\
\hline $\mathbf{P}_{4} \times \mathbf{P}_{5}$ & 2.23 & 0.66 & $3.71 * *$ & 0.064 & -0.20 & 2.61 & -0.50 & 0.089 & -0.66 \\
\hline $\mathbf{P}_{4} \times \mathbf{P}_{6}$ & 1.26 & $-1.60 * *$ & -0.74 & 0.657 & $0.48^{*}$ & 0.86 & $-3.70 * *$ & $1.61 *$ & 1.58 \\
\hline $\mathbf{P}_{5} \times \mathbf{P}_{6}$ & 2.04 & $-1.26^{*}$ & $-7.27 * *$ & -0.01 & -0.10 & -1.01 & 0.04 & 0.59 & 0.75 \\
\hline SE & 1.40 & 0.54 & 0.66 & 0.15 & 0.22 & 2.01 & 1.23 & 0.68 & 0.91 \\
\hline
\end{tabular}

Chl: Teneur en chlorophylle (unité Spad); DE: Date d'épiaison (jours); HT: Hauteur de la plante (cm); NE: Nombre d'épi par plante; NGE: Nombre de grains par épi; PMG: Poids de mille grains (g); RDT: Rendement en grains par plante (g); IR: Indice de récolte $(\%) ; *$ et $*$ : Effets significatifs au seuil de $1 \%$ et $5 \%$, respectivement; SE: Erreur standard.

Les $\mathrm{F}_{2}$ Zenati-Bouteille/Flamengo x Guemgoum Rkhem $\left(\mathrm{P}_{2} \times \mathrm{P}_{6}\right)$, Mexicali 75 x Guemgoum Rkhem $\left(\mathrm{P}_{3} \times \mathrm{P}_{6}\right)$ et Ofanto $\mathrm{x}$ Guemgoum Rkhem $\left(\mathrm{P}_{4} \times \mathrm{P}_{6}\right)$ présentent des effets d'ASC 
significatifs et positifs pour la hauteur, le nombre de grains par épi, le nombre d'épis et le rendement par plante. La sélection tardive est à suggérer pour les caractères des populations $\mathrm{F}_{2}$ montrant des effets d'ASC significatifs.

\subsection{Relations entre les caractères}

Considéré comme étant complexe, sous contrôle polygénique et fortement influencé par l'environnement, la sélection basée sur le rendement est peu efficace. L'étude des corrélations génétiques fournit des informations clés sur la contribution relative des différents caractères dans l'élaboration du rendement en grains et aide à définir celui qui pourrait être utilisé comme critère de sélection pour identifier des lignées supérieures. La sélection indirecte sur la base des caractères qui présentent une héritabilité élevée associée à une forte corrélation avec le rendement est extrêmement recommandée. La matrice des corrélations entre les caractères mesurés en $F_{2}$ met en évidence de nombreuses relations significatives (Table 6). Le poids de mille grains est positivement et significativement corrélé avec la date d'épiaison, la hauteur de la végétation et la longueur de l'épi mais négativement lié au nombre d'épis par plante, au nombre de grains par épi et à l'indice de récolte.

Table 6. Coefficients de corrélations génotypiques entre les caractères mesurés en $\mathrm{F}_{2}$.

\begin{tabular}{|c|c|c|c|c|c|c|c|c|c|}
\hline & Chl & DE & HT & $\mathbf{L E}$ & NE & NGE & PMG & RDT & IR \\
\hline Chl & 1.000 & & & & & & \multicolumn{2}{|c|}{$r_{5 \%}=0.432$} & \\
\hline DE & $-0.810 * *$ & 1.000 & & & & & \multicolumn{2}{|c|}{$\mathrm{r}_{1 \%}=0.548$} & \\
\hline HT & $-0.637 * *$ & 0.790 ** & 1.000 & & & & & & \\
\hline $\mathbf{L E}$ & $-0.534 *$ & $0.584 * *$ & $0.694 * *$ & 1.000 & & & & & \\
\hline $\mathbf{N E}$ & $0.502 *$ & $-0.579 * *$ & -0.364 & -0.326 & 1.000 & & & & \\
\hline NGE & 0.045 & -0.217 & $-0.457^{*}$ & 0.236 & -0.054 & 1.000 & & & \\
\hline PMG & -0.422 & $0.727^{* *}$ & $0.857 * *$ & $0.561^{* *}$ & $-0.594 * *$ & $-0.485^{*}$ & 1.000 & & \\
\hline RDT & 0.172 & -0.232 & 0.084 & 0.370 & 0.373 & 0.281 & -0.035 & 1.000 & \\
\hline HI & $0.809 * *$ & $-0.868 * *$ & $-0.772 * *$ & -0.378 & 0.387 & 0.499* & $-0.619 * *$ & 0.322 & 1.000 \\
\hline
\end{tabular}

Chl: Teneur en chlorophylle (unité Spad); DE: Date d'épiaison (jours); HT: Hauteur de la plante (cm); NE: Nombre d'épi par plante; NGE: Nombre de grains par épi; PMG: Poids de mille grains (g); RDT: Rendement en grains par plante (g); IR: Indice de récolte $(\%) ; *$ et *: corrélation significatifs au seuil de $1 \%$ et $5 \%$, respectivement.

De cette étude, il ressort que la durée de la phase végétative semble être associée d'une part à un poids de mille grains et une hauteur élevés et d'autre part à une production réduite du nombre de talles fertiles et de la fertilité des épis. Ces résultats corroborent avec ceux obtenus 
par Abbessene et al. [33] et Hannachi et al. [34]. Des corrélations hautement significatives sont enregistrées entre l'indice de récolte et la teneur en chlorophylle, la date d'épiaison, la hauteur des plantes et le poids de mille grains. L'augmentation de l'indice de récolte demeure la modification majeure intervenue en termes de progrès génétique réalisé chez les variétés de blé modernes par rapport aux variétés anciennes pendant la révolution verte [35]. Cette augmentation s'est traduite par une amélioration spectaculaire des rendements céréaliers. Aucune corrélation statistiquement significative n'est, par contre, observée entre le rendement et le reste des caractères étudiés. Ce résultat prouve la difficulté d'identifier un/des critère (s) de sélection indirecte autre que le rendement final. Néanmoins, les corrélations positives entre le rendement et le nombre d'épis d'une part et négatives entre le rendement et la date d'épiaison d'autre part, même avec faible amplitude, suggère que ces deux variables devraient être considérées comme étant de bons indicateurs d'une productivité élevée. Dans ce contexte, plusieurs auteurs dont Fellahi et al. [36] sur blé tendre et Guendouz et al. [37] sur blé dur attribuent à ces deux caractères une importance primordiale au regard de leurs contributions majeures au rendement sous conditions pluviales où les stress postfloraison sont omniprésents.

\section{CONCLUSION}

Les résultats montrent que les actions géniques de nature aussi bien additives que non additives sont impliquées dans le contrôle génétique des caractères étudiés, avec prépondérance des actions géniques de nature additive. Par conséquent, la sélection précoce pour améliorer la teneur en chlorophylle, la précocité à l'épiaison, la hauteur de la végétation, le nombre de grains par épi et le poids de mille grains pourrait être efficace. Quant à la sélection du rendement, elle doit être faite sur des générations tardives plus homogènes et ce, après fixation des effets géniques de dominance. La sélection à l'intérieure des croisements Waha $x$ Ofanto $\left(\mathrm{P}_{1} \times \mathrm{P}_{4}\right)$, Zenati-Bouteille/Flamengo $\mathrm{x}$ Gta/durum $\left(\mathrm{P}_{2} \times \mathrm{P}_{5}\right)$ et Ofanto $\mathrm{x}$ Guemgoum Rkhem $\left(\begin{array}{lll}\mathrm{P}_{4} & \mathrm{x} & \mathrm{P}_{6}\end{array}\right)$ pourraient apporter des améliorations de plusieurs caractéristiques à la fois dont le rendement suite aux effets positifs et significatifs enregistrés pour les ASC chez ces populations. Sous des conditions semi-arides, la sélection de génotypes 
précoces ayant un nombre de talles fertiles élevé pourrait améliorer le rendement en grains chez le matériel génétique évalué.

\section{REFERENCES}

[1] Nazco R, Villegas D, Ammar K, Pena R J, Moragues M, and Roy C. Can Mediterranean durum wheat landraces contribute to improved grain quality attributes in modern cultivars? Euphytica, 2012, 185: 1-17.

[2] Fellahi Z, Hannachi A, Rabti A, Benmahammed A, et Bouzerzour H. Analyse graphique d'un croisement Lignées x Testeurs de blé tendre. Séminaire national sur la Problématique et les enjeux de l'agriculture Algérienne INRA Algérie- Université Abou Bakr Belkaid-Tlemcen - 11 Avril 2016.

[3] Singh B N, Vishwakarma S R, and Singh V K. Character association and path analysis in elite lines of wheat (Triticum aestivum L.). J. Plant Arch., 2010, 10(2): 845-847.

[4] Farshadfar E, Hasheminasab H, and Yaghotipoor A. Estimation of combining ability and gene action for improvement drought tolerance in bread wheat (Triticum aestivum L.) using GGE biplot techniques. J. Agric. Sci., 2012, 4(9): 1-10.

[5] Adel M M, and Ali E A. Gene action and combining ability in a six parent diallel cross of wheat. Asian J. Crop Sci., 2013, 5(1): 14-23.

[6] Singh R K, and Chaudhary B D. Biometrical methods in quantitative genetic analysis, Kalyani Publishers, New Delhi, India, 1985. 318 pages

[7] Gallais A. Génétique quantitative et méthodes de sélection chez les espèces autopolyploïdes. Editions Quae, 2003, 515 pages.

[8] Fellahi Z, Hannachi A, Bouzerzour H, and Boutekrabt A. Line $\times$ Tester Mating Design Analysis for Grain Yield and Yield Related Traits in Bread Wheat (Triticum aestivum L.). Int. J. Agron., 2013, Article ID 201851, 9 pages.

[9] Hannachi A, Fellahi Z, Bouzerzour H, and Boutekrabt A. Diallel-cross analysis of grain yield and stress tolerance-related traits under semi-arid conditions in Durum wheat (Triticum durum Desf.). Elect. J. Plant Breed., 2013, 4(1): 1027-1033.

[10] Nduwumuremyi A, Tongoona P, and Habimana S. Mating designs: helpful tool for 
quantitative plant breeding analysis. J. Plant Breed. Genet., 2013, 1(3), 117-129.

[11] Hannachi A. Analyse diallèle de quelques caractères associes à l'adaptation du blé dur (Triticum durum Desf.) au climat semi-aride. Mémoire de Magister, Université Saad Dahlab, Blida, 2013, 106 pages.

[12] Kashif M, and Khaliq I. Mechanism of genetic control of some quantitative traits in bread wheat. Pak. J. Biol. Sci., 2003, 6(18): 1586-1590.

[13] Mahmood N, Chowdhry M A, and Kashif M. Genetic analysis of some physio-morphic traits of wheat under drought condition (Triticum aestivum L.). Pak. J. Genet. Breed., 2003, 57: 385-391.

[14] Rathod S K K, Dobariya L, Jivani L L, and Ponkia H P. Analysis for test weight, protein content and grain yield in six crosses of bread wheat (Triticum aestivum L.). Advances in Plant Sci., 2008, 21(1): 99-101.

[15] Rabbani G M, Munir S U, Ajmal F, Hassan G, Shabbir, and Mahmood A. Inheritance of yield attributes in bread wheat under irrigated and rainfed conditions. Sarhad J. Agric., 2009, 25(3): 429-438.

[16] Farooq J, Khaliq I, Khan A S, and Pervez M A. Studying the genetic mechanism of some yield contributing traits in wheat. (Triticum aestivum L). Int. J. Agri. Biol., 2010, 12: 241-246.

[17] Fellahi Z, Hannachi A, Bouzerzour H, and Benbelkacem A. Inheritance Pattern of Metric Characters Affecting Grain Yield in Two Bread Wheat (Triticum aestivum L.) Crosses Under Rainfed Conditions. 2015, Jordan J. Biol. Sci., 8(3): 175-181.

[18] Steel R G D, and Torrie J. Principles and Procedures of Statistics, A Biometric Approach. McGraw-Hill Book Company, NY., 1980, 633 pages.

[19] Griffing B. Concepts of general and specific combining ability in relation to diallel crossing systems. Aust. J. Biol. Sci., 1956, 9: 463-493.

[20] Cruz C D. GENES - a software package for analysis in experimental statistics and quantitative. Acta Sci. Agron., 2013, 35(3): 271-276.

[21] Baker R J, Issues in diallel analysis. Crop Sci., 1978, 18(4): 533-536.

[22] Teklewold A, Becker H C. Heterosis and combining ability in a diallel cross of Ethiopian mustard inbred lines. Crop Sci., 2005, 45: 2629-2635. 
[23] Iqbal M, Navabi A, Salmon D F, Yang R C, and Spaner D. A genetic examination of early flowering and maturity in Canadian spring wheats. Canadian J. Plant Sci., 2006, 86(4): 995-1004.

[24] Shahid N, Abduslam K, and Zuflqar A. Combining ability analysis for yield and yield contributing traits in bread wheat. J. Agric. Social Sci., 2015, 2: 129-132.

[25] Bouzerzour H, and Djekoun A. Inheritance of grain yield and grain yield components in barley. Rachis, 1998, 16: 9-16.

[26] Falconer D S. Introduction to quantitative genetics. Longman Scientific \& Technical (Ed.), London, 1981, 340 pages.

[27] Dabholkar A R. Elements of bio metrical genetics. Revised and enlarged edition. New Delhi: Concept Publishing Company, 1999, 493 pages.

[28] Al-Tabbal J A, and Al-Fraihat A H. Heritability Studies of Yield and Yield Associated Traits in Wheat Genotypes, J. Agric. Sci., 2012, 4(4): 11-22.

[29] Riaz U D, Subhani G M, Ahmad N, Hussain M, and UR Rehman A. Effect of temperature on development and grain formation in spring wheat. Pak. J. Bot., 2010, 42(2): 899-906.

[30] Ali Y, Atta B M, Akhter J, Monneveux P, and Lateef Z. Genetic variability, association and diversity studies in wheat (Triticum aestivum L.) germplasm. Pak. J. Bot., 2008, 40(5): 2087-2097.

[31] Mather K, and Jinks J L. Biometrical Genetics. London, Chapman \& Hall, 1971, 231 pages.

[32] Bensemane L, et Bouzerzour H. Rythme de développement des variétés contrastées de blé dur (Triticum durum Desf). I- Analyse de la croissance-développement. Rec. Agr., 2006, 18: 30-52.

[33] Abbassene F, Bouzerzour H, et Hachemi L. Phénologie et production du blé dur (T. dirrum Desf.) en zone semi-aride d'altitude. Annales Agronomrques. INA, 1997, 18(1 et 2), 24-36.

[34] Hannachi A, Fellahi Z, Bouzerzour H, and Boutkrabt A. Correlation, Path Analysis and Stepwise Regression in Durum Wheat (Triticum durum Desf.) under Rainfed Conditions. 
2013, J. Agr. Sust., 3(2): 122-131.

[35] Gallais A. Comprendre l'amélioration des plantes : Enjeu, methodes, objectifs et critères de sélection. Editions Quae, 2015, 231 p.

[36] Fellahi Z, Hannachi A, Guendouz A, Bouzerzour H, and Boutekrabt A. Genetic variability, heritability and association studies in bread wheat (Triticum aestivum L.) genotypes. Elec. J. Plant Breed., 2013, 4(2): 1161-1166.

[37] Guendouz A, Djoudi M., Guessoum S, Maamri K, Hannachi A, Fellahi Z, and Hafsi M. Genotypic and phenotypic correlations among yield and yield components in Durum Wheat (Triticum durum Desf.) under different water treatments in Eastern Algeria. Ann. Res. \& Review in Biol., 2014, 4(2): 432-442.

\section{How to cite this article:}

Hannachi A, Fellahi Z, Rabti A, Guendouz A, and Bouzerzour H. Combining ability and gene action estimates for some yield attributes in durum wheat (Triticum turgidium L. var. durum). J. Fundam. Appl. Sci., 2017, 9(3), 1519-1534. 